\title{
Integrated Application Scheme of Substation Intelligent Primary Equipments
}

\author{
Ran LIU ${ }^{1, a}$, Dayong $Y U^{1, b}$, Dongxue $\mathrm{LI}^{1, \mathrm{c}}$, Zhengchang JIAO ${ }^{2, \mathrm{~d}}$ and Tao LIU ${ }^{1, \mathrm{e}}$ \\ ${ }^{1}$ State Grid Liaoning Electric Power Company Limited Economic Research Institute, Shenyang, \\ Liaoning Province, 110015, China \\ ${ }^{2}$ Keya college, Shenyang university of chemical technology, Shenyang, Liaoning Province, 110167, \\ China \\ apsincos@163.com, bydy_jyy@In.sgcc.com.cn, ' Idx_jyy@In.sgcc.com.cn, djiaozhc@163.com, elt_jy \\ y@In.sgcc.com.cn
}

Keywords: Intelligent substation; Integrated application; Primary equipment

\begin{abstract}
There is a clear boundary between primary and secondary devices in traditional substation. The intelligent demand of intelligent substation is analyzed. According to the design idea and method of intelligent substation, the integrated application scheme of the disconnecting circuit breaker, intelligent transformer, electronic transducer, switch cabinet and intelligent capacitor technology in substation is put forward.
\end{abstract}

\section{Introduction}

Traditional substation is made up of primary and secondary devices. Primary equipments mainly include transformers, gas insulated switchgear, circuit breakers, mutual inductors, etc.. Secondary equipments include protective devices, measure and control devices, metering devices, monitoring devices and other electronic devices. These devices are distributed in a substation, and there is a clear boundary between primary and secondary devices. Intelligent substation is characterized by a high degree of reliability, interactivity and high degree of integration. At present, in intelligent substations having been put into operation, the integration of primary device ontology and on-line monitoring sensor has been achieved [1]. Devices for protection, measurement and control devices, and merging unit, intelligent terminal are integrated in the use of different voltage levels substations. Future intelligent devices will be highly integrated, to break the boundaries between an existing primary and secondary devices, and intelligent components can have the functions of protection, measurement, control, measurement and monitoring of the state, with organic integration with primary devices. In order to meet the needs of the construction of the Smart Substation and intelligent high voltage equipment, the intelligent substation primary equipment needs is studied, and an integrated application scheme of substation intelligent devices is put forward.

\section{Intelligence of integrated disconnecting circuit breaker}

The disconnecting circuit breaker is referred to as DCB. When the contact is in the position of the switch, the switch is required to meet the requirements of the switch [2]. Its shape size is consistent with the ordinary circuit breaker. It has three working positions: closing position, opening position, switch position. When the break of the breaker reaches the pressure level of the isolating switch, it has the function of isolating switch. The function is realized by the interrupter contacts of the breaker. Electronic Transducer can be implemented with DCB or combined with an integrated installation. DCB combines the traditional circuit breaker with the function of isolating switch. The dynamic and static contact of the equipment is protected in the SF6 arc-chute, which has the dual function of the circuit breaker and the isolating switch. Intelligent DCB is composed of isolated circuit breaker body, intelligent components and sensors. Various sensors and operating mechanisms are installed on the circuit breaker body. Sensors are responsible for monitoring the state parameters of the circuit breaker, which can be transmit to an intelligent component by means of digital or analog, and then 
through the intelligent components reported to the intelligent substation upper level systems. Therefore, the upper level system of the intelligent substation can realize the observation of the state of DCB, and then support the optimal operation of the smart grid. At the same time, the intelligent substation upper system can send instructions to the intelligent components according to the results of the observations. After logical judgment, the intelligent component sends out the instruction to the operating mechanism to perform the operation, in order to achieve the purpose of the optimization control of the intelligent power grid [3].

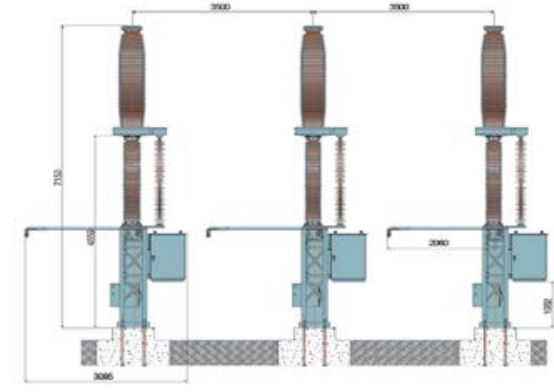

(a) $220 \mathrm{kV}$

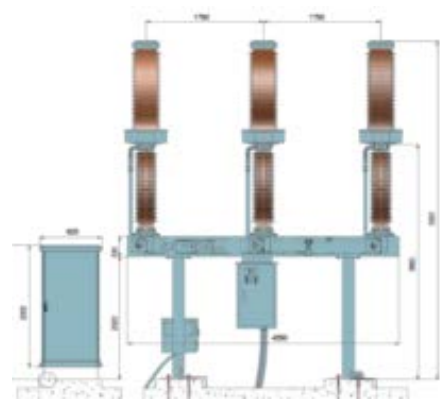

(b) $110 \mathrm{kV}$

Fig.1. Intelligent DCB assembly drawing

\section{Intelligent transformer}

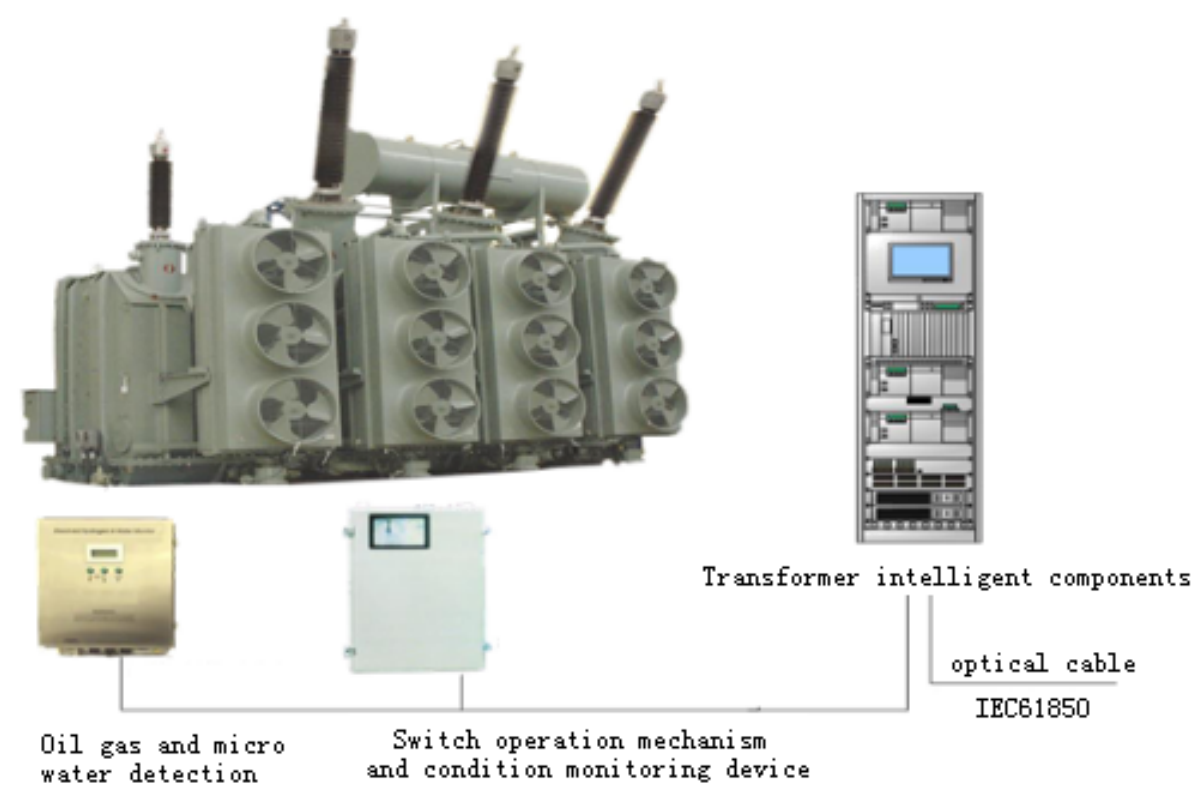

Fig.2. Intelligent transformer structure diagram

The implementation of intelligent transformer is implanting sensor into the traditional transformer, and adding intelligent components [4]. In use of the digital and networking technology, and with transformer state sensor and Instruction execution element, Visualization of transformer state and network and automation of control functions can be realized. The integration design of the intelligent components and the main body of the transformer is needed. In the process level and bay level, all functions such as measurement, inspection, control and protection need to be assumed. $220 \mathrm{kV}$ intelligent transformer mainly includes: transformer body, sensor, and intelligent component cabinet. With the integration of sensor and transformer, the requirement of the auto detection is fully considered in the initial stage of the design. This will not only improve the integrity of the device, it will improve the quality of self testing also, to protect the safety of the host equipment. Oil and gas monitoring is realized using electrochemical method, with sensor installed in the transformer oil tank bottom. Continuous on-line monitoring is made about the concentrations and trends of mixed gas of $\mathrm{H} 2, \mathrm{CO}, \mathrm{C} 2 \mathrm{H} 4, \mathrm{C} 2 \mathrm{H} 2$ in transformer oil. Intelligent component cabinet is the collection of several 
IED, intelligent components, installed in the transformer body, and taking the measurement, control and monitoring functions related to transformer equipment. Transformer intelligent unit is mainly used for on-line continuous monitoring and comprehensive evaluation of transformer working state. The integrated assessment and diagnosis of the transformer operation condition is accomplished by combining the embedded evaluation and diagnosis technology. Communication with the station control device will be realized through the optical fiber Ethernet interface.

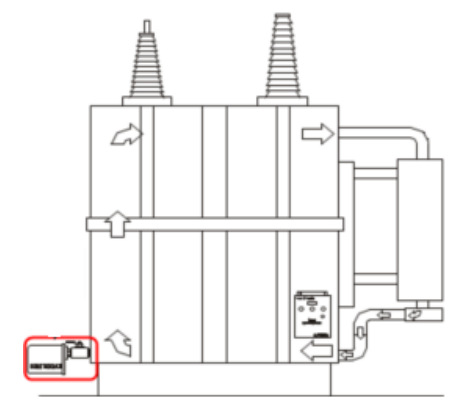

Fig.3. Mounting position of oil gas monitoring sensor

Tab.1. Function table of transformer intelligent units

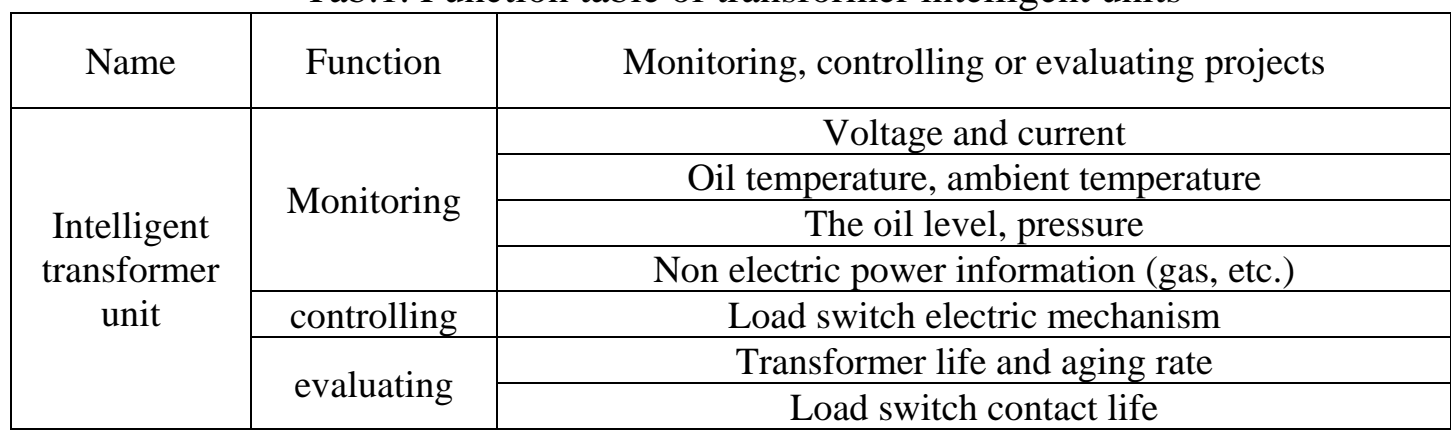

\section{Electronic transducer}

Electronic Transducer is based on the principle of the photoelectric technology. It is the essential equipment of the intelligent power grid. The high voltage side and the low voltage side of the Electronic Transducer are connected by optical fiber, and the optical fiber has good insulation performance, which can ensure that the primary and secondary circuit can be completely isolated. There is no danger because of the high voltage caused by the open circuit or high current generated by the short circuit. Electronic Transducer has large transient response range, high measuring accuracy and wide frequency response range. Electronic Transducer does not use oil insulation, so it is small with light weight. The higher the voltage level is, the more obvious the benefit will be, and it is not easy to produce flammable and explosive hazard while filled with oil. Electronic Transducer output digital quantity, so it is easy to communicate with the control system [5].

\section{$10 \mathrm{kV}$ intelligent switchgear}

$10 \mathrm{kV}$ intelligent switchgear is highly integrated and intelligent device. From the control and protection function is concerned, it should have a certain intelligence, that is, to make the intelligent switch cabinet according to the state of the switch and the power grid operation mode, automatically select the best mode to control and protect. In terms of structure, the switch cabinet equipment should be fully modular and standardized, so the intelligent switch cabinet is not only hardware, but also need measurement, relay protection, measurement, control and other functions. Intelligent component is the core of intelligent switch cabinet, which is integrated with the functions of protection, measurement and control, measurement and fault recording. Features of integrated and large-scale integrated circuits are used to make intelligent products more compact. According to different control objects, the switch cabinet is divided into line, transformer, capacitor and other units, which monitoring and protection of the object is different. But they have the same hardware, so they 
can be interchangeable. With different software, it can be formed different functions of the measurement and control unit, so as to reduce the spare parts. Smart component with measurement, control, protection, communication and other functions is installed on the switch cabinet, so only a small amount of cable can complete the wiring work. And all kinds of functions can be realized by software, which simplifies the work of maintenance and calibration.

\section{The intelligent assembling capacitor device}

The intelligent high voltage assembling capacitor device is composed of assembling capacitor, a series reactor, a discharge coil, an arrester and an intelligent component. The built-in UHF sensor, with resistant to strong magnetic field, high frequency resistance, microwave field resistance, good explosion proof, which anti vibration and anti shock performance is good, is easy on quantitative analysis. The intelligent set of high voltage assembling capacitor covers an area of about 1/2 of the ordinary type of capacitor. Intelligent component cabinet is the core of the intelligent assembly type of parallel capacitor. It contains the intelligent unit, the protection unit, the temperature, the pressure monitoring, the Bureau, the oil gas monitoring IED device, and the built-in ultra high frequency PD sensor. The capacitor protection is put in the $10 \mathrm{kV}$ switch cabinet. The protection unit, temperature and pressure monitoring, bureau, oil and gas monitoring IED devices is used to collect and monitor the status of a device, and then upload it to the intelligent unit. The operation state of the capacitor is determined by data analysis, so as to ensure its reliable operation.

\section{Conclusion}

According to the design idea and method of intelligent substation, the integrated application scheme of the DCB, the intelligent transformer, the electronic transformer, the intelligent switch cabinet and the intelligent capacitor in the substation is put forward. After the equipment integration and optimization design, the operation reliability of the substation can be improved obviously, and the area of the substation and the construction area have been greatly reduced.

\section{References}

[1] SONG Xuankun, LI Jingru, XIAO Zhihong, LIN Hongyu, LI Zhenyu, ZOU Guohui, HUANG Baoying, LIN Yong. Overall Design Scheme for New Generation Intelligent Substation [J]. Electric Power Construction, 2012(11):1-6.

[2] Huang Yangqi, Lu Xinyi, Liu Nian, Zhang Jianhua. Analysis on Reliability and Sensitivity of Main Electrical Connection for New Generation Smart Substation with Disconnecting Circuit Breaker[J]. Research on Development. 2014, 6:10-14.

[3] CHEN Xiaojie. Techniques of $500 \mathrm{kV}$ smart unattended substation[J]. Electric Power Automation Equipment, 2011(31):149-152.

[4] GAO Xiang, ZHANG Peichao. Main features and key technologies of digital substation[J]. Power System Technology, 2006(23):67-71.

[5] MA Shi-hai, JIN Zhi-xin, GAO Yang. Discussion on intelligent substation technology system[J]. JOURNAL OF SHENYANG INSTITUTE OF ENGINEERING(NATURAL SCIENCE). 2010, 06(4):333-336. 